\title{
Geometry of product complex Cartan manifolds
}

\author{
Nicoleta Aldea and Gheorghe Munteanu
}

\begin{abstract}
In this paper we consider the product of two complex Cartan manifolds, the outcome being a class of product complex Cartan spaces. Then, we study the relationships between the geometric objects of a product complex Cartan space and its components, (e.g. Chern-Cartan complex nonlinear connection, Cartan tensors). By means of these, we establish the necessary and sufficient conditions under which a product complex Cartan space is Landsberg-Cartan or Berwald-Cartan or it has some other properties.
\end{abstract}

\section{Introduction}

The geometry of the holomorphic cotangent bundle, endowed with a complex homogeneous Hamiltonian on the fibre, is known as complex Cartan geometry, $[10,9,14,5,15]$. It is a natural development, into the complex context, of the remarkable results from real Hamilton and Cartan geometries, mainly due to R. Miron, $[13,12,7]$. Of course, there are some formal similarities with the real approach, but the differences between real and complex cases have the upper hand.

The study of the complex Cartan spaces has been justified by the existence of a pseudo-distance on the holomorphic cotangent bundle, pointed out by S. Kobayashi in [10].

Key Words: complex Cartan metric, product manifold, Landsberg- and Berwald-Cartan metrics.

2010 Mathematics Subject Classification: Primary 53C60; Secondary 53B40.

Received: 2 May, 2014.

Accepted: 28 June, 2014. 
In complex Cartan geometry there are not so many known examples of complex Cartan metrics. Besides the significant dual Kobayashi metric characterized by J. Faran in [9], there are two rather trivial classes of complex Cartan metrics: the complex Cartan metrics which come from Hermitian metrics on the base manifold (so called the purely Hermitian metrics in [14]) and the locally Minkowski complex metrics, [14]. Recently, in the papers, [5, 6], we initiated the study of some classes of complex Cartan spaces: LandsbergCartan, Kähler-Cartan, Berwald-Cartan, etc, which are exemplified by complex Cartan-Randers metrics. Nevertheless, any other examples are welcome.

Twisted product structures are widely used in geometry to build examples of Finsler manifolds, [11]. The product of two complex Finsler manifolds is approached by W. Zhicheng and C. Zhong in [18]. Therefore, it is natural to extend this construction for complex Cartan spaces. Using some ideas from the complex Finsler case [18], our aim in the present paper is to introduce and study product complex Cartan spaces.

The paper is organized as follows. After we make a short survey of complex Cartan spaces (Section 2), in Section 3 we construct a class of complex Cartan spaces, on the product $M:=M_{1} \times M_{2}$, where $\left(M_{1}, \mathcal{C}_{1}\right)$ and $\left(M_{2}, \mathcal{C}_{2}\right)$ are two complex Cartan manifolds, (Theorem 3.1). We find that the study of a such product complex Cartan space is reduced to the study of its components $\left(M_{1}, \mathcal{C}_{1}\right)$ and $\left(M_{2}, \mathcal{C}_{2}\right)$. More exactly, the non-vanishing components of ChernCartan complex nonlinear connection associated to the product complex Cartan space are the Chern-Cartan complex nonlinear connection of $\left(M_{1}, \mathcal{C}_{1}\right)$ and $\left(M_{2}, \mathcal{C}_{2}\right)$, respectively.

Based on this, in section 4 we obtain the conditions under which the product complex Cartan space is Kähler-Cartan or weakly Kähler-Cartan, (Theorem 4.1). Also, we prove that the product complex Cartan space is LandsbergCartan or Berwald-Cartan if and only if its components $\left(M_{1}, \mathcal{C}_{1}\right)$ and $\left(M_{2}, \mathrm{C}_{2}\right)$ have similar properties, (Theorem 4.2). Finally, in Theorem 4.3 are given the necessary and sufficient conditions that the product complex Cartan space to be purely Hermitian.

\section{Preliminaries}

Geometry of real and complex Finsler spaces is already one classic today, $([1,8,16,2,14,17,3,4]$, etc.). Also, the study of Cartan spaces (real and complex) is enthralling, ([12, 13, 7, 14, 6], etc.). In this section we will give some preliminaries about complex Cartan geometry with Chern-Cartan and Berwald complex linear connections. We will set the basic notions (for more see $[14,6,5])$.

Let $M$ be a $n$ - dimensional complex manifold and $z=\left(z^{k}\right)_{k=\overline{1, n}}$ be 
complex coordinates in a local chart. The complexified of the real tangent bundle $T_{C} M$ splits into the sum of holomorphic tangent bundle $T^{\prime} M$ and its conjugate $T^{\prime \prime} M$. The dual of $T^{\prime} M$ is denoted by $T^{\prime *} M$. On the manifold $T^{* *} M$, a point $u^{*}$ is characterized by the coordinates $u^{*}=(z, \zeta)=\left(z^{k}, \zeta_{k}\right)_{k=\overline{1, n}}$, and a change of these has the form $z^{\prime k}=z^{\prime k}(z)$ and $\zeta_{k}^{\prime}=\frac{\partial^{*} z^{j}}{\partial z^{\prime k}} \zeta_{j}, \operatorname{rank}\left(\frac{\partial^{*} z^{\prime k}}{\partial z^{l}}\right)=n$.

Definition 2.1. A complex Cartan space is a pair $(M, \mathcal{C})$, where $\mathcal{C}: T^{*} M \rightarrow$ $\mathbb{R}^{+},\left(\mathbb{R}^{+}:=[0, \infty)\right)$, is a continuous function satisfying the conditions:

i) $H:=\mathcal{C}^{2}$ is smooth on $\widetilde{T^{*} M}:=T^{* *} M \backslash\{0\}$;

ii) $\mathrm{C}(z, \zeta) \geq 0$, the equality holds if and only if $\zeta=0$;

iii) $\mathrm{C}(z, \lambda \zeta)=|\lambda| C(z, \zeta)$ for $\forall \lambda \in \mathbb{C}$;

iv) the Hermitian matrix $\left(h^{\bar{j} i}(z, \zeta)\right)$ is positive definite, where $h^{\bar{j} i}:=\frac{\partial^{2} H}{\partial \zeta_{i} \partial \bar{\zeta}_{j}}$ is the fundamental metric tensor.

Equivalently, the condition $i v$ ) means that the indicatrix is strongly pseudoconvex.

Consequently, from $i i i)$ we have $\frac{\partial H}{\partial \zeta_{k}} \zeta_{k}=\frac{\partial H}{\partial \bar{\zeta}_{k}} \bar{\zeta}_{k}=H, \frac{\partial h^{\bar{j} i}}{\partial \zeta_{k}} \zeta_{k}=\frac{\partial h^{\bar{j} i}}{\partial \bar{\zeta}_{k}} \bar{\zeta}_{k}=0$ and $H=h^{\bar{j} i} \bar{\zeta}_{j} \zeta_{i}$. An usual example of complex Cartan space is so called purely Hermitian complex Cartan space, this means that $h^{\bar{j} i}=h^{\bar{j} i}(z)$.

The geometry of a complex Cartan space consists in the study of the geometric objects of the complex manifold $T^{*} M$ endowed with the Hermitian metric structure defined by $h^{\bar{j} i}$. So, the first step is the study of sections in the complexified tangent bundle of $T^{*} M$, which is decomposed in the sum $T_{C}\left(T^{*} M\right)=T^{\prime}\left(T^{* *} M\right) \oplus T^{\prime \prime}\left(T^{* *} M\right)$.

Let $V T^{* *} M \subset T^{\prime}\left(T^{* *} M\right)$ be the vertical bundle, which has the vertical distribution $V_{u^{*}}\left(T^{* *} M\right)$, locally spanned by $\left\{\frac{\partial}{\partial \zeta_{k}}\right\}$. A complex nonlinear connection, briefly (c.n.c.), on $T^{* *} M$ is a supplementary subbundle in $T^{\prime}\left(T^{* *} M\right)$ of $V\left(T^{*} M\right)$, i.e., $T^{\prime}\left(T^{*} M\right)=H\left(T^{* *} M\right) \oplus V\left(T^{* *} M\right)$. The horizontal distribution $H_{u^{*}}\left(T^{* *} M\right)$ is locally spanned by $\left\{\frac{\delta^{*}}{\delta z^{j}}\right\}$, where $\frac{\delta^{*}}{\delta z^{k}}=\frac{\partial^{*}}{\partial z^{k}}+N_{j k} \frac{\partial}{\partial \zeta_{j}}$ and functions $N_{j k}$ are the coefficients of the (c.n.c.) on $T^{* *} M$. The pair $\left\{\delta_{k}^{*}:=\right.$ $\left.\frac{\delta^{*}}{\delta z^{k}}, \dot{\partial}^{k}:=\frac{\partial}{\partial \zeta_{k}}\right\}$ will be called the adapted frame of the (c.n.c.), which obey the change rules $\delta_{k}^{*}=\frac{\partial^{*} z^{\prime j}}{\partial z^{k}} \delta_{j}^{* \prime}$ and $\dot{\partial}^{k}=\frac{\partial^{*} z^{\prime k}}{\partial z^{j}} \dot{\partial}^{\prime j}$. By conjugation everywhere we have obtained an adapted frame $\left\{\delta_{\bar{k}}^{*}, \dot{\partial}^{\bar{k}}\right\}$ on $T_{u^{*}}^{\prime \prime}\left(T^{* *} M\right)$. The dual adapted frames are $\left\{d^{*} z^{k}, \delta \zeta_{k}=d \zeta_{k}-N_{k j} d z^{j}\right\}$ and $\left\{d^{*} \bar{z}^{k}, \delta \bar{\zeta}_{k}\right\}$.

A Hermitian connection $D \Gamma$, of $(1,0)$ - type is so called Chern-Cartan connection (cf. [14]), and it is locally given by the following coefficients

$$
N_{j i}=-h_{j \bar{k}} \frac{\partial^{*} h^{\bar{k} l}}{\partial z^{i}} \zeta_{l} ; H_{j k}^{i}:=h^{\bar{m} i}\left(\delta_{k}^{*} h_{j \bar{m}}\right) ; V_{j}^{i k}:=-h_{j \bar{m}}\left(\dot{\partial}^{k} h^{\bar{m} i}\right),
$$


and $H_{\bar{j} k}^{\bar{\imath}}=V_{\bar{j}}^{\bar{\imath} k}=0$, where here and hereinafter $\delta_{k}^{*}$ is the adapted frame of the Chern-Cartan (c.n.c.), $h_{j \bar{k}} h^{\bar{k} l}=\delta_{j}^{l}$ and $D_{\delta_{k}^{*}} \delta_{j}^{*}=H_{j k}^{i} \delta_{i}, D_{\delta_{k}^{*}} \dot{\partial}^{i}=$ $-H_{j k}^{i} \dot{\partial}^{j}, D_{\dot{\partial}^{k}} \delta_{j}^{*}=V_{j}^{i k} \delta_{i}^{*}, D_{\dot{\partial}^{k}} \dot{\partial}^{i}=-V_{j}^{i k} \dot{\partial}^{j}$, etc. Moreover, we have

$$
H_{j k}^{i}=\dot{\partial}^{i} N_{j k} ; H_{j k}^{i} \zeta_{i}=N_{j k},
$$

and Chern-Cartan connection $D \Gamma:=\left(N_{j i}, H_{j k}^{i}, 0, V_{j}^{i k}, 0\right)$ is $h^{*}-$ and $v^{*}-$ metrical, (for more details see [14]).

Further on, in order to simplify the writing, we use a bar over indices to denote the complex conjugation of the variables or of the frames, e.g., $\zeta_{\bar{k}}:=\bar{\zeta}_{k}$ or $\dot{\partial}^{\bar{k}}:=\overline{\dot{\partial}^{k}}$, in some places.

In [5] we investigated some classes of complex Cartan spaces. A complex Cartan space $(M, \mathcal{C})$ is called Kähler-Cartan iff $T_{j k}^{* i} \zeta_{i}=0$ and weakly KählerCartan iff $T_{j k}^{* i} \zeta_{i} \zeta^{j}=0$, where $T_{j k}^{* i}:=H_{j k}^{i}-H_{k j}^{i}$ is the $h^{*}$-torsion and $\zeta^{j}:=$ $h^{\bar{m} j} \zeta_{\bar{m}}$. A necessary and sufficient condition for Kähler-Cartan property is $N_{j k}=N_{k j}$, [6]. The space $(M, \mathcal{C})$ is called Berwald-Cartan iff the coefficients $H_{j k}^{i}$ depend only on the position $z$. This condition is equivalent with the holomorphicity in $\zeta$ of the local coefficients $N_{j i}$ of Chern-Cartan (c.n.c.), (i.e. $\left.\dot{\partial}^{i} N_{j k}=0\right)$.

Another complex linear connection associated to the Chern-Cartan (c.n.c.) is one of Berwald type $B \Gamma:=\left(N_{j i}, B_{j k}^{i}:=\dot{\partial}^{i} N_{j k}, B_{\bar{j} k}^{\bar{\imath}}:=\left(\dot{\partial}^{\bar{\imath}} N_{k s}\right) \zeta^{s} \zeta_{\bar{j}}, 0,0\right)$, which is neither $h^{*}-$ nor $v^{*}-$ metrical, but it has $B_{j k}^{i}=H_{j k}^{i}$. When $B \Gamma$ is $h^{*}$ - metrical, $(M, \mathcal{C})$ is called complex Landsberg-Cartan space in [5]. This is equivalent with either $B \Gamma$ is of $(1,0)$ - type or the functions $\mathbf{L}_{k}^{\bar{\imath}}:=\left(\dot{\partial}^{\bar{\imath}} N_{k s}\right) \zeta^{s}$ vanish identically. Note that any complex Berwald-Cartan space is a complex Landsberg-Cartan space and any Landsberg-Cartan space with weakly KählerCartan property is a Kähler-Cartan space, (Theorem 3.3 from [5]). Also, any complex Kähler-Cartan space is a complex Landsberg-Cartan space.

Moreover, on a complex Cartan space, the complex Cartan tensors have the expressions: $V^{\bar{m} i k}=\dot{\partial}^{k} h^{\bar{m} i}$ and its complex conjugate $V^{\bar{m} \bar{\imath} k}=\dot{\partial}^{\bar{\imath}} h^{\bar{m} k}$. The homogeneity of $C$ leads to $V^{\bar{m} i k} \zeta_{i}=0$ and $V^{\bar{m} \bar{\imath} k} \zeta_{\bar{\imath}}=0$. Moreover, it is know that $V^{\bar{m} i k}=0$ iff $\mathcal{C}$ is purely Hermitian.

\section{Product complex Cartan spaces}

In this section, we turn to account some ideas from [18], to construct the complex Cartan product metrics. We consider two complex Cartan spaces $\left(M_{1}, \mathcal{C}_{1}\right)$ and $\left(M_{2}, \mathcal{C}_{2}\right)$ of complex dimensions $m$ and $n$, respectively. The complex coordinate in the local charts on $M_{1}$ and on $M_{2}$ are denoted by $\left(z^{\alpha}\right)_{\alpha=\overline{1, m}}$ and $\left(z^{\alpha^{\prime}}\right)_{\alpha^{\prime}=\overline{m+1, m+n}}$, respectively. On the manifolds $T^{\prime *} M_{1}$ and $T^{*} M_{2}$ the points 
are characterized by the coordinates $\left(z^{\alpha}, \zeta_{\alpha}\right)_{\alpha=\overline{1, m}}$ and $\left(z^{\alpha^{\prime}}, \zeta_{\alpha^{\prime}}\right)_{\alpha^{\prime}=\overline{m+1, m+n}}$. Here and further, we use the Greek letters $\alpha, \beta, \gamma, \ldots$ and $\alpha^{\prime}, \beta^{\prime}, \gamma^{\prime}, \ldots$ for the ranges of indices $\overline{1, m}$ and $\overline{m+1, m+n}$, respectively. Also, for the complex Cartan spaces $\left(M_{1}, \mathrm{C}_{1}\right)$ and $\left(M_{2}, \mathrm{C}_{2}\right)$ the fundamental metric tensors are denoted by

$$
k^{\bar{\beta} \alpha}:=\frac{\partial^{2} K}{\partial \zeta_{\alpha} \partial \bar{\zeta}_{\beta}} \text { and } l^{\bar{\beta}^{\prime} \alpha^{\prime}}:=\frac{\partial^{2} L}{\partial \zeta_{\alpha^{\prime}} \partial \bar{\zeta}_{\beta^{\prime}}},
$$

respectively, where $K:=\mathcal{C}_{1}^{2}$ and $L:=\mathcal{C}_{2}^{2}$. Moreover, making the notations $k^{\alpha}:=\dot{\partial}^{\alpha} K$ and $l^{\alpha^{\prime}}:=\dot{\partial}^{\alpha^{\prime}} L$, the homogeneity of both $K$ and $L$ gives $k^{\alpha} \zeta_{\alpha}=$ $K, k^{\bar{\beta} \alpha} \zeta_{\bar{\beta}} \zeta_{\alpha}=K, l^{\alpha^{\prime}} \zeta_{\alpha^{\prime}}=L$ and $l^{\bar{\beta}^{\prime} \alpha^{\prime}} \zeta_{\bar{\beta}^{\prime}} \zeta_{\alpha^{\prime}}=L$.

The product $M:=M_{1} \times M_{2}$ is a $m+n-$ dimensional complex manifold and $z=\left(z^{k}\right)_{k=1, m+n}$ are the local complex coordinates. Since there is a natural isomorphism between $T^{* *} M$ and $T^{* *} M_{1} \oplus T^{* *} M_{2},\left(z^{i}, \zeta_{i}\right)_{i=\overline{1, m+n}}$ are the local complex coordinate on the complex manifold $T^{* *} M$. We use the Latin letters $i, j, k, \ldots$ for the ranges of indices $\overline{1, m+n}$. We consider a function $\mathrm{e}: \widetilde{T^{*} M_{1}} \times \widetilde{T^{* *} M_{2}} \subset \widehat{T^{*} M} \rightarrow \mathbb{R}^{+}$given by

$$
\mathcal{C}(z, \zeta)=\sqrt{f\left(K\left(z^{\alpha}, \zeta_{\alpha}\right), L\left(z^{\alpha^{\prime}}, \zeta_{\alpha^{\prime}}\right)\right)}
$$

where $f$ is a real valued smooth function, $f: \mathbb{R}^{+} \times \mathbb{R}^{+} \rightarrow \mathbb{R}^{+}$, which satisfies the conditions:

a) $f(K, L)=0$ if and only if $(K, L)=(0,0)$;

b) $f(\lambda K, \lambda L)=\lambda f(K, L)$, for $\forall \lambda \geq 0$;

c) $f_{K} \neq 0, f_{L} \neq 0$ and $f_{K} f_{L}-f f_{K L} \neq 0$ for $K, L \neq 0$, where $f_{K}:=\frac{\partial f}{\partial K}$, $f_{L}:=\frac{\partial f}{\partial L}, f_{K L}:=\frac{\partial^{2} f}{\partial K \partial L}=f_{L K}$.

The homogeneity condition $b$ ) implies:

$K f_{K}+L f_{L}=f ; K f_{K K}+L f_{K L}=0 ; K f_{K L}+L f_{L L}=0 ; f_{K L}^{2}=f_{K K} f_{L L}$

The function $\mathcal{C}$ defined in (3.1) can be a complex Cartan metric. Indeed, the properties of the function $f$ asserts the conditions $i)-i i i)$ from Definition 2.1. With the notations $H:=\mathcal{C}^{2}=f\left(K\left(z^{\alpha}, \zeta_{\alpha}\right), L\left(z^{\alpha^{\prime}}, \zeta_{\alpha^{\prime}}\right)\right), \zeta^{\alpha}:=\dot{\partial}^{\alpha} H$, $\zeta^{\alpha^{\prime}}:=\dot{\partial}^{\alpha^{\prime}} H$ and $\zeta^{i}:=\dot{\partial}^{i} H$ we find the explicit form of the Hermitian matrix

$$
\left(h^{\bar{j} i}(z, \zeta)\right)_{i, j=\overline{1, m+n}}=\left(\frac{\partial^{2} H}{\partial \zeta_{i} \partial \bar{\zeta}_{j}}\right)_{i, j=\overline{1, m+n}}=\left(\begin{array}{cc}
\left(h^{\bar{\beta} \alpha}\right) & \left(h^{\bar{\beta}^{\prime} \alpha}\right) \\
\left(h^{\bar{\beta} \alpha^{\prime}}\right) & \left(h^{\bar{\beta}^{\prime} \alpha^{\prime}}\right)
\end{array}\right),
$$

where

$$
\begin{aligned}
& \left(h^{\bar{\beta} \alpha}\right):=\frac{\partial^{2} H}{\partial \zeta_{\alpha} \partial \bar{\zeta}_{\beta}}=f_{K K}\left(k^{\alpha} k^{\bar{\beta}}\right)+f_{K}\left(k^{\bar{\beta} \alpha}\right) ; \\
& \left(h^{\bar{\beta}^{\prime} \alpha}\right):=\frac{\partial^{2} H}{\partial \zeta_{\alpha} \partial \bar{\zeta}_{\beta^{\prime}}}=f_{K L}\left(k^{\alpha} l^{\bar{\beta}^{\prime}}\right) ;
\end{aligned}
$$




$$
\begin{aligned}
& \left(h^{\bar{\beta} \alpha^{\prime}}\right):=\frac{\partial^{2} H}{\partial \zeta_{\alpha^{\prime}} \partial \zeta_{\beta}}=f_{K L}\left(l^{\alpha^{\prime}} k^{\bar{\beta}}\right) \\
& \left(h^{\bar{\beta}^{\prime} \alpha^{\prime}}\right):=\frac{\partial^{2} H}{\partial \zeta_{\alpha^{\prime}} \partial \bar{\zeta}_{\beta^{\prime}}}=f_{L L}\left(l^{\alpha^{\prime}} l^{\bar{\beta}^{\prime}}\right)+f_{L}\left(l^{\bar{\beta}^{\prime} \alpha^{\prime}}\right) .
\end{aligned}
$$

The same technique as in [18] lead us to the following result:

Theorem 3.1. The pair $\left(M:=M_{1} \times M_{2}, \mathcal{C}\right)$, with $\mathcal{C}$ defined by (3.1), is a complex Cartan space, called product complex Cartan space, if and only if the function $f$ satisfies the conditions:

$$
\begin{aligned}
f_{K} & >0 ; f_{L}>0 ; f_{K} f_{L}-f f_{K L}>0 ; \\
f_{K}+K f_{K K} & >0 ; f_{L}+L f_{L L}>0 .
\end{aligned}
$$

Moreover,

i) $\operatorname{det}\left(h^{\bar{j} i}\right)=f_{K}^{m-1} f_{L}^{n-1}\left(f_{K} f_{L}-f f_{K L}\right) \operatorname{det}\left(k^{\bar{\beta} \alpha}\right) \operatorname{det}\left(l^{\bar{\beta}^{\prime} \alpha^{\prime}}\right)$;

ii) The inverse of $\left(h^{\bar{j} i}\right)$ is

$$
h_{i \bar{j}}=\left(\begin{array}{cc}
h_{\alpha \bar{\beta}} & h_{\alpha \bar{\beta}^{\prime}} \\
h_{\alpha^{\prime} \bar{\beta}} & h_{\alpha^{\prime} \bar{\beta}^{\prime}}
\end{array}\right),
$$

where

$h_{\alpha \bar{\beta}}:=\frac{1}{f_{K}}\left[k_{\alpha \bar{\beta}}-\frac{f_{L} f_{K K}}{A} \zeta_{\alpha} \zeta_{\bar{\beta}}\right] ; h_{\alpha \bar{\beta}^{\prime}}:=-\frac{f_{K L}}{A} \zeta_{\alpha} \zeta_{\bar{\beta}^{\prime}} ;$

$h_{\alpha^{\prime} \bar{\beta}}:=-\frac{f_{K L}}{A} \zeta_{\alpha^{\prime}} \zeta_{\bar{\beta}} ; h_{\alpha^{\prime} \bar{\beta}^{\prime}}:=\frac{1}{f_{L}}\left[l_{\alpha^{\prime} \bar{\beta}^{\prime}}-\frac{f_{K} f_{L L}}{A} \zeta_{\alpha^{\prime}} \zeta_{\bar{\beta}^{\prime}}\right]$;

$A:=f_{K} f_{L}+L f_{K} f_{L L}+K f_{L} f_{K K}$ and $\left(k_{\alpha \bar{\beta}}\right)$ and $\left(l_{\alpha^{\prime} \bar{\beta}^{\prime}}\right)$ are the inverse of $k^{\bar{\beta} \alpha}$ and $l^{\bar{\beta}^{\prime} \alpha^{\prime}}$, respectively.

Now, we consider the local coefficients of Chern-Cartan (c.n.c.) associated to the complex Cartan metrics $\mathcal{C}_{1}$ and $\mathcal{C}_{2}$ :

$$
\hat{N}_{\alpha \beta}=-k_{\alpha \bar{\delta}} \frac{\partial^{*} k^{\bar{\delta} \gamma}}{\partial z^{\beta}} \zeta_{\gamma} ; \check{N}_{\alpha^{\prime} \beta^{\prime}}=-l_{\alpha^{\prime} \bar{\delta}^{\prime}} \frac{\partial^{*} l_{\bar{\delta}^{\prime} \gamma^{\prime}}}{\partial z^{\beta^{\prime}}} \zeta_{\gamma^{\prime}}
$$

Next, by means of these, a technical computation yields the expressions of the local coefficients of Chern-Cartan (c.n.c.), associated to product complex Cartan space $\left(M:=M_{1} \times M_{2}, \mathcal{C}\right)$.

Lemma 3.1. Let $\left(M:=M_{1} \times M_{2}, \mathcal{C}\right)$ be the product complex Cartan space, with $\mathcal{C}$ defined by (3.1). Then, the local coefficients of Chern-Cartan (c.n.c.) corresponding to $\left(M:=M_{1} \times M_{2}, \mathcal{C}\right)$ are given by following

$$
\left(N_{j i}\right)=\left(\begin{array}{cc}
N_{\alpha \beta} & N_{\alpha^{\prime} \beta} \\
N_{\alpha \beta^{\prime}} & N_{\alpha^{\prime} \beta^{\prime}}
\end{array}\right)
$$

where $N_{\alpha \beta}=\hat{N}_{\alpha \beta}, N_{\alpha^{\prime} \beta^{\prime}}=\breve{N}_{\alpha^{\prime} \beta^{\prime}}$ and $N_{\alpha^{\prime} \beta}=N_{\alpha \beta^{\prime}}=0$. 
Proof. It results by a trivial computation, taking into account (3.2) and

$$
N_{j i}=-h_{j \bar{k}} \frac{\partial^{*}}{\partial z^{i}}\left(\dot{\partial}^{\bar{k}} H\right)=-h_{j \bar{\gamma}} \frac{\partial^{*}}{\partial z^{i}}(\dot{\partial} \bar{\gamma} \bar{\gamma} H) h_{j \bar{\gamma}^{\prime}} \frac{\partial^{*}}{\partial z^{i}}\left(\dot{\partial}^{\bar{\gamma}^{\prime}} H\right),
$$

where

$$
\begin{aligned}
& \dot{\partial} \bar{\gamma} H=f_{K} k^{\bar{\gamma}}, \dot{\partial} \bar{\gamma}^{\prime} H=f_{L} l^{\bar{\gamma}^{\prime}} \\
& \frac{\partial^{*}}{\partial z^{\beta}}\left(\dot{\partial}^{\bar{\gamma}} H\right)=f_{K K} \frac{\partial^{*} K}{\partial z^{\beta}} k^{\bar{\gamma}}+f_{K} \frac{\partial^{*} k^{\bar{\gamma} \delta}}{\partial z^{\beta}} \zeta_{\delta} ; \\
& \frac{\partial^{*}}{\partial z^{\beta}}\left(\dot{\partial}^{\gamma^{\prime}} H\right)=f_{K L} \frac{\partial^{*} K}{\partial z^{\beta}} l \bar{\gamma}^{\prime} ; \\
& \frac{\partial^{*}}{\partial z^{\beta^{\prime}}}(\dot{\partial} \bar{\gamma} H)=f_{K L} \frac{\partial^{*} L}{\partial z^{\beta^{\prime}}} k^{\bar{\gamma}} \\
& \frac{\partial^{*}}{\partial z^{\beta^{\prime}}}\left(\dot{\partial} \bar{\gamma}^{\prime} H\right)=f_{L L} \frac{\partial^{*} L}{\partial z^{\beta^{\prime}}} l^{\bar{\gamma}^{\prime}}+f_{L} \frac{\partial^{*} l^{\bar{\gamma}^{\prime} \delta \prime}}{\partial z^{\beta^{\prime}}} \zeta_{\delta^{\prime}} \text {. } \\
& \text { Indeed, we have } \\
& N_{\alpha \beta}=-h_{\alpha \bar{\gamma}} \frac{\partial^{*}}{\partial z^{\beta}}(\dot{\partial} \bar{\gamma} H)-h_{\alpha \bar{\gamma}^{\prime}} \frac{\partial^{*}}{\partial z^{\beta}}\left(\dot{\partial}^{\bar{\gamma}^{\prime}} H\right) \\
& =-\frac{1}{f_{K}}\left[k_{\alpha \bar{\gamma}}-\frac{f_{L} f_{K K}}{A} \zeta_{\alpha} \zeta_{\bar{\gamma}}\right]\left[f_{K K} \frac{\partial^{*} K}{\partial z^{\beta}} k^{\bar{\gamma}}+f_{K} \frac{\partial^{*} k^{\bar{\gamma} \delta}}{\partial z^{\beta}} \zeta_{\delta}\right]+\frac{f_{K L}^{2}}{A} \frac{\partial^{*} K}{\partial z^{\beta}} \zeta_{\alpha} \zeta_{\bar{\gamma}^{\prime}} l^{\bar{\gamma}^{\prime}}= \\
& \hat{N}_{\alpha \beta} \text {. }
\end{aligned}
$$

Analogous, we obtain the other components of the matrix $\left(N_{j i}\right)$.

\section{Some classes of product complex Cartan spaces}

The expression of the Chern-Cartan (c.n.c.) of a product complex Cartan space is very convenient, it makes that some properties of both complex Cartan manifolds $\left(M_{1}, \mathfrak{C}_{1}\right)$ and $\left(M_{2}, \mathfrak{C}_{2}\right)$ to be devolved on the product complex Cartan manifold and, conversely.

Theorem 4.1. Let $(M, \mathcal{C})$ be the product complex Cartan space of the complex Cartan spaces $\left(M_{1}, \mathcal{C}_{1}\right)$ and $\left(M_{2}, \mathcal{C}_{2}\right)$, with $M:=M_{1} \times M_{2}$ and $\mathcal{C}$ defined by (3.1).Then,

i) $(M, \mathrm{C})$ is weakly Kähler-Cartan if and only if both spaces $\left(M_{1}, \mathrm{C}_{1}\right)$ and $\left(M_{2}, \mathrm{C}_{2}\right)$ are weakly Kähler-Cartan.

ii) $(M, \mathcal{C})$ is Kähler-Cartan if and only if both spaces $\left(M_{1}, \mathcal{C}_{1}\right)$ and $\left(M_{2}, \mathrm{C}_{2}\right)$ are Kähler-Cartan.

Proof. i) Corresponding to the product complex Cartan space $(M, \mathcal{C})$ we have

$$
T_{j k}^{* i} \zeta_{i} \zeta^{j}=\left(T_{j \beta}^{* i} \zeta_{i} \zeta^{j}, T_{j \beta^{\prime}}^{* i} \zeta_{i} \zeta^{j}\right)
$$

and

$$
T_{j k}^{* i} \zeta_{i} \zeta^{j}=\left(N_{j k}-N_{k j}\right) \zeta^{j}=\left(N_{\alpha k}-N_{k \alpha}\right) \zeta^{\alpha}+\left(N_{\alpha^{\prime} k}-N_{k \alpha^{\prime}}\right) \zeta^{\alpha^{\prime}} .
$$

Using Lemma 3.1, it results 


$$
\begin{aligned}
& T_{j \beta}^{* i} \zeta_{i} \zeta^{j}=\left(N_{\alpha \beta}-N_{\beta \alpha}\right) \zeta^{\alpha}+\left(N_{\alpha^{\prime} \beta}-N_{\beta \alpha^{\prime}}\right) \zeta^{\alpha^{\prime}} \\
& =f_{K}\left(\hat{N}_{\alpha \beta}-\hat{N}_{\beta \alpha}\right) k^{\alpha}=f_{K} \hat{T}_{\alpha \beta}^{* \gamma} \zeta_{\gamma} \zeta^{\alpha} \text { and } \\
& T_{j \beta^{\prime}}^{* i} \zeta_{i} \zeta^{j}=\left(N_{\alpha \beta^{\prime}}-N_{\beta^{\prime} \alpha}\right) \zeta^{\alpha}+\left(N_{\alpha^{\prime} \beta^{\prime}}-N_{\beta^{\prime} \alpha^{\prime}}\right) \zeta^{\alpha^{\prime}} \\
& =f_{L}\left(\breve{N}_{\alpha^{\prime} \beta^{\prime}}-\breve{N}_{\beta^{\prime} \alpha^{\prime}}\right) l^{\alpha^{\prime}}=f_{L} \breve{T}_{\alpha^{\prime} \beta^{\prime}}^{* \gamma^{\prime}} \zeta_{\gamma^{\prime}} \zeta^{\alpha^{\prime}}
\end{aligned}
$$

where the functions $\hat{T}_{\alpha \beta}^{* \gamma} \zeta_{\gamma} \zeta^{\alpha}$ and $\breve{T}_{\alpha^{\prime} \beta^{\prime}}^{* \gamma^{\prime}} \zeta_{\gamma^{\prime}} \zeta^{\alpha^{\prime}}$ contain the horizontal torsion coefficients associated to the spaces $\left(M_{1}, \mathcal{C}_{1}\right)$ and $\left(M_{2}, \mathcal{C}_{2}\right)$, respectively. Due the above relations, we obtain that $T_{j k}^{* i} \zeta_{i} \zeta^{j}=(0,0)$ iff $\hat{T}_{\alpha \beta}^{* \gamma} \zeta_{\gamma} \zeta^{\alpha}=0$ and $\breve{T}_{\alpha^{\prime} \beta^{\prime}}^{* \gamma^{\prime}} \zeta_{\gamma^{\prime}} \zeta^{\alpha^{\prime}}=0$ which prove i).

ii) Using again Lemma 3.1 , for the product space $(M, \mathcal{C})$ we have $\left(T_{j k}^{* i} \zeta_{i}\right)=$ $\left(N_{j k}-N_{k j}\right)=\left(\begin{array}{cc}\hat{N}_{\alpha \beta}-\hat{N}_{\beta \alpha} & 0 \\ 0 & \breve{N}_{\alpha^{\prime} \beta^{\prime}}-\breve{N}_{\beta^{\prime} \alpha^{\prime}}\end{array}\right)$, which attest that $N_{j k}=N_{k j}$ iff $\hat{N}_{\alpha \beta}=\hat{N}_{\beta \alpha}$ and $\breve{N}_{\alpha^{\prime} \beta^{\prime}}=\breve{N}_{\beta^{\prime} \alpha^{\prime}}$.

Theorem 4.2. Let $(M, \mathcal{C})$ be the product complex Cartan space of the complex Cartan spaces $\left(M_{1}, \mathcal{C}_{1}\right)$ and $\left(M_{2}, \mathcal{C}_{2}\right)$, with $M:=M_{1} \times M_{2}$ and $\mathcal{C}$ defined by (3.1). Then,

i) $(M, \mathrm{C})$ is Landsberg-Cartan if and only if both spaces $\left(M_{1}, \mathrm{C}_{1}\right)$ and $\left(M_{2}, \mathrm{C}_{2}\right)$ are Landsberg-Cartan.

ii) $(M, \mathcal{C})$ is Berwald-Cartan if and only if both spaces $\left(M_{1}, \mathrm{C}_{1}\right)$ and $\left(M_{2}, \mathcal{C}_{2}\right)$ are Berwald-Cartan.

Proof. i) The functions $\mathbf{L}_{k}^{\bar{\imath}}$ associated to the product space $(M, \mathcal{C})$ can be written as $\mathbf{L}_{k}^{\bar{z}}=\left(\dot{\partial}^{\bar{\imath}} N_{k s}\right) \zeta^{s}=\left(\dot{\partial}^{\bar{\imath}} N_{k \alpha}\right) \zeta^{\alpha}+\left(\dot{\partial}^{\bar{\imath}} N_{k \alpha^{\prime}}\right) \zeta^{\alpha^{\prime}}$ and $\left(\mathbf{L}_{k}^{\bar{z}}\right)=\left(\begin{array}{cc}\mathbf{L}_{\gamma^{\prime}}^{\bar{\beta}} & \mathbf{L}_{\gamma^{\prime}}^{\bar{\beta}} \\ \mathbf{L}_{\gamma}^{\bar{\beta}^{\prime}} & \mathbf{L}_{\gamma^{\prime}}^{\beta^{\prime}}\end{array}\right)$.

Taking into account Lemma 3.1, it get

$$
\begin{aligned}
& \mathbf{L}_{\gamma}^{\bar{\beta}}=\left(\dot{\partial}^{\bar{\beta}} N_{\gamma \alpha}\right) \zeta^{\alpha}+\left(\dot{\partial}^{\bar{\beta}} N_{\gamma \alpha^{\prime}}\right) \zeta^{\alpha^{\prime}}=f_{K}\left(\dot{\partial}_{\gamma \alpha}^{\bar{\beta}} \hat{N}\right) k^{\alpha}=f_{K} \hat{\mathbf{L}}_{\gamma}^{\bar{\beta}} ; \\
& \mathbf{L}_{\gamma^{\prime}}^{\bar{\beta}}=\left(\dot{\partial}^{\bar{\beta}} N_{\gamma^{\prime} \alpha}\right) \zeta^{\alpha}+\left(\dot{\partial}^{\bar{\beta}} N_{\gamma^{\prime} \alpha^{\prime}}\right) \zeta^{\alpha^{\prime}}=0 ; \\
& \mathbf{L}_{\gamma}^{\bar{\beta}^{\prime}}=\left(\dot{\partial}^{\bar{\beta}^{\prime}} N_{\gamma \alpha}\right) \zeta^{\alpha}+\left(\dot{\partial}^{\bar{\beta}^{\prime}} N_{\gamma \alpha^{\prime}}\right) \zeta^{\alpha^{\prime}}=0 \text { and } \\
& \mathbf{L}_{\gamma^{\prime}}^{\bar{\beta}^{\prime}}=\left(\dot{\partial}^{\bar{\beta}^{\prime}} N_{\gamma^{\prime} \alpha}\right) \zeta^{\alpha}+\left(\dot{\partial}^{\bar{\beta}^{\prime}} N_{\gamma^{\prime} \alpha^{\prime}}\right) \zeta^{\alpha^{\prime}}=f_{L}\left(\dot{\partial}^{\bar{\beta}^{\prime}} \breve{N}_{\gamma^{\prime} \alpha^{\prime}}\right) l^{\alpha^{\prime}}=f_{L} \breve{\mathbf{L}}_{\gamma^{\prime}}^{\bar{\beta}^{\prime}} \text {, where the }
\end{aligned}
$$
functions $\hat{\mathbf{L}}_{\gamma}^{\bar{\beta}}:=\left(\dot{\partial}^{\bar{\beta}} \hat{N}_{\gamma \alpha}\right) \zeta^{\alpha}$ and $\breve{\mathbf{L}}_{\gamma^{\prime}}^{\bar{\beta}^{\prime}}:=\left(\dot{\partial}_{\gamma^{\prime} \alpha^{\prime}}^{\bar{\beta}^{\prime}} \breve{N}\right) \zeta^{\alpha^{\prime}}$ correspond to the spaces $\left(M_{1}, \mathcal{C}_{1}\right)$ and $\left(M_{2}, \mathcal{C}_{2}\right)$, respectively. Thus, $\mathbf{L}_{k}^{\bar{\imath}}=0$ iff $\hat{\mathbf{L}}_{\gamma}^{\bar{\beta}}=0$ and $\breve{\mathbf{L}}_{\gamma^{\prime}}^{\bar{\beta}^{\prime}}=0$.

ii) Using Lemma 3.1 it implies $\left(\dot{\partial}^{\bar{\imath}} N_{j k}\right)=\left(\begin{array}{cc}\dot{\partial} \bar{\gamma} \hat{N}_{\alpha \beta} & 0 \\ 0 & \dot{\partial}^{\bar{\gamma}^{\prime}}{\stackrel{N}{\alpha^{\prime} \beta^{\prime}}}^{\prime}\end{array}\right)$ and so, $\dot{\partial}^{\bar{\imath}} N_{j k}=0$ iff $\hat{N}_{\alpha \beta}$ and $\breve{N}_{\alpha^{\prime} \beta^{\prime}}$ are holomorphic in $\zeta_{\bar{\gamma}}$ and $\zeta_{\bar{\gamma}^{\prime}}$, respectively.

Corollary 4.1. Let $(M, \mathcal{C})$ be the product complex Cartan space of the complex Cartan spaces $\left(M_{1}, \mathrm{C}_{1}\right)$ and $\left(M_{2}, \mathrm{C}_{2}\right)$, with $M:=M_{1} \times M_{2}$ and $\mathcal{C}$ defined by 
(3.1). If one of the spaces $\left(M_{1}, \mathrm{C}_{1}\right)$ and $\left(M_{2}, \mathrm{C}_{2}\right)$ is Kähler-Cartan and the other is Berwald-Cartan, then $(M, \mathrm{C})$ is Landsberg-Cartan.

Proof. Under our assumptions, the spaces $\left(M_{1}, \mathrm{C}_{1}\right)$ and $\left(M_{2}, \mathrm{C}_{2}\right)$ are Landsberg-Cartan. Thus, applying Theorem 4.2 i), it results our claim.

Further on, we find the explicit form of the complex Cartan tensors $V^{\bar{j} i k}$ associated to the product complex Cartan space $\left(M:=M_{1} \times M_{2}, \mathcal{C}\right)$, with $\mathrm{C}$ defined by (3.1). The complex Cartan tensors corresponding to the metrics $\mathrm{C}_{1}$ and $\mathrm{C}_{2}$ are following

$$
\hat{V}^{\bar{\beta} \alpha \gamma}:=\dot{\partial}^{\gamma} k^{\bar{\beta} \alpha} \text { and } \breve{V}^{\bar{\beta}^{\prime} \alpha^{\prime} \gamma^{\prime}}:=\dot{\partial} \gamma^{\prime} l^{\bar{\beta}^{\prime} \alpha^{\prime}},
$$

respectively, and their complex conjugates. A direct computation leads to the result.

Lemma 4.1. Let $(M, \mathrm{C})$ be the product complex Cartan space of the complex Cartan spaces $\left(M_{1}, \mathrm{C}_{1}\right)$ and $\left(M_{2}, \mathrm{C}_{2}\right)$, with $M:=M_{1} \times M_{2}$ and $\mathrm{C}$ defined by (3.1). Then,

$$
V^{\bar{j} i k}=\left(V^{\bar{\beta} \alpha \gamma}, V^{\bar{\beta} \alpha \gamma^{\prime}}, V^{\bar{\beta}^{\prime} \alpha \gamma}, V^{\bar{\beta}^{\prime} \alpha \gamma^{\prime}}, V^{\bar{\beta} \alpha^{\prime} \gamma}, V^{\bar{\beta} \alpha^{\prime} \gamma^{\prime}}, V^{\bar{\beta}^{\prime} \alpha^{\prime} \gamma}, V^{\bar{\beta}^{\prime} \alpha^{\prime} \gamma^{\prime}}\right),
$$

where

$$
\begin{aligned}
& V^{\bar{\beta} \alpha \gamma}=\dot{\partial}^{\gamma} h^{\bar{\beta} \alpha}=f_{K K K} k^{\bar{\beta}} k^{\alpha} k^{\gamma} \\
& +f_{K K}\left(\hat{V}^{\bar{\delta} \alpha \gamma} \zeta_{\bar{\delta}} k^{\bar{\beta}}+k^{\bar{\beta} \gamma} k^{\alpha}+k^{\bar{\beta} \alpha} k^{\gamma}\right)+f_{K} \hat{V}^{\bar{\beta} \alpha \gamma} \text {; } \\
& V^{\bar{\beta} \alpha \gamma^{\prime}}=\dot{\partial} \gamma^{\prime} h^{\bar{\beta} \alpha}=\left(f_{K K L} k^{\bar{\beta}} k^{\alpha}+f_{K L} k^{\bar{\beta} \alpha}\right) l^{\gamma^{\prime}} ; \\
& V^{\bar{\beta}^{\prime} \alpha \gamma}=\dot{\partial}^{\gamma} h^{\bar{\beta}^{\prime} \alpha}=\left(f_{K K L} k^{\alpha} k^{\gamma}+f_{K L} \hat{V}^{\bar{\delta} \alpha \gamma} \zeta_{\bar{\delta}}\right) l^{\bar{\beta}^{\prime}} ; \\
& V^{\bar{\beta}^{\prime} \alpha \gamma^{\prime}}=\dot{\partial} \gamma^{\prime} h^{\bar{\beta}^{\prime} \alpha}=\left(f_{K L L} l^{\bar{\beta}^{\prime}} l^{\gamma^{\prime}}+f_{K L} l^{\bar{\beta} \gamma^{\prime}}\right) k^{\alpha} ; \\
& V^{\bar{\beta} \alpha^{\prime} \gamma}=\dot{\partial}^{\gamma} h^{\bar{\beta} \alpha^{\prime}}=\left(f_{K K L} k^{\bar{\beta}} k^{\gamma}+f_{K L} k^{\bar{\beta} \gamma}\right) l^{\alpha^{\prime}} ; \\
& V^{\bar{\beta} \alpha^{\prime} \gamma^{\prime}}=\dot{\partial} \gamma^{\prime} h^{\bar{\beta} \alpha^{\prime}}=\left(f_{K L L} l^{\alpha} l^{\gamma}+f_{K L} \breve{V}^{\bar{\delta}^{\prime} \alpha^{\prime} \gamma^{\prime}} \zeta_{\bar{\delta}^{\prime}}\right) k^{\bar{\beta}} ; \\
& V^{\bar{\beta}^{\prime} \alpha^{\prime} \gamma}=\dot{\partial}^{\gamma} h^{\bar{\beta}^{\prime} \alpha^{\prime}}=\left(f_{K L L} l^{\bar{\beta}^{\prime}} l^{\alpha^{\prime}}+f_{K L} l^{\bar{\beta}^{\prime} \alpha^{\prime}}\right) k^{\gamma} ; \\
& V^{\bar{\beta}^{\prime} \alpha^{\prime} \gamma^{\prime}}=\dot{\partial} \gamma^{\prime} h^{\bar{\beta}^{\prime} \alpha^{\prime}}=f_{L L L} l^{\bar{\beta}^{\prime}} l^{\alpha^{\prime}} l^{\gamma^{\prime}} \\
& +f_{L L}\left(\breve{V}^{\bar{\delta}^{\prime} \alpha^{\prime} \gamma^{\prime}} \zeta_{\bar{\delta}^{\prime}} l^{\bar{\beta}^{\prime}}+l^{\bar{\beta}^{\prime} \gamma^{\prime}} l^{\alpha^{\prime}}+l^{\bar{\beta}^{\prime} \alpha^{\prime}} l^{\gamma^{\prime}}\right)+f_{L} \breve{V}^{\bar{\beta}^{\prime} \alpha^{\prime} \gamma^{\prime}} .
\end{aligned}
$$

Theorem 4.3. Let $(M, \mathcal{C})$ be the product complex Cartan space of the complex Cartan spaces $\left(M_{1}, \mathrm{C}_{1}\right)$ and $\left(M_{2}, \mathrm{C}_{2}\right)$, with $M:=M_{1} \times M_{2}$ and $\mathrm{C}$ defined by (3.1).Then, $\mathcal{C}$ is a purely Hermitian metric if and only if $\mathrm{C}_{1}$ and $\mathrm{C}_{2}$ are purely Hermitian metrics and

$$
f(K, L)=c_{1} K+c_{2} L, c_{1}, c_{2}>0 .
$$


Proof. We suppose that $\mathcal{C}$ is a purely Hermitian metric. Then, $V^{\bar{j} i k}=0$ and contracting in (4.3) with suitable combinations of $\zeta_{\bar{*}} \zeta_{*} \zeta_{*}$, where $* \epsilon$ $\left\{\alpha, \beta, \gamma, \alpha^{\prime}, \beta^{\prime}, \gamma^{\prime}\right\}$, we obtain the following system of equations

$$
\begin{aligned}
K f_{K K K}+2 f_{K K} & =0 ; L f_{L L L}+2 f_{L L}=0 \\
f_{K K L} & =f_{K L L}=f_{K L}=0 .
\end{aligned}
$$

Now, the homogeneity condition $b$ ) implies: $f_{K K}=0$ and $f_{L L}=0$ which give $f(K, L)=c_{1} K+c_{2} L, c_{1}, c_{2}>0$. Replacing this in (4.3), it results $f_{K} \hat{V}^{\bar{\beta} \alpha \gamma}=f_{L} \breve{V}^{\bar{\beta}^{\prime} \alpha^{\prime} \gamma^{\prime}}=0$. Since $f_{K}>0$ and $f_{L}>0$, the last conditions leads to $\hat{V}^{\bar{\beta} \alpha \gamma}=\breve{V}^{\bar{\beta}^{\prime} \alpha^{\prime} \gamma^{\prime}}=0$, i.e. the metrics $\mathcal{C}_{1}$ and $\mathcal{C}_{2}$ are purely Hermitian.

Conversely, if $\mathcal{C}_{1}$ and $\mathcal{C}_{2}$ are purely Hermitian metrics and $f(K, L)=c_{1} K+$ $c_{2} L, c_{1}, c_{2}>0$, then (4.3) is reduced to $V^{\bar{j} i k}=0$ which completes the proof.

Since any purely Hermitian complex Cartan metric is itself one BerwaldCartan, by Theorem 4.2 ii) we have justified the following result.

Corollary 4.2. Let $(M, \mathcal{C})$ be the product complex Cartan space of the complex Cartan spaces $\left(M_{1}, \mathcal{C}_{1}\right)$ and $\left(M_{2}, \mathcal{C}_{2}\right)$, with $M:=M_{1} \times M_{2}$ and $\mathcal{C}$ defined by (3.1). If $\mathrm{C}_{1}$ and $\mathrm{C}_{2}$ are purely Hermitian metrics, then $(M, \mathcal{C})$ is a BerwaldCartan space.

If we choose the function $f: \mathbb{R}^{+} \times \mathbb{R}^{+} \rightarrow \mathbb{R}^{+}$to be

$$
f(K, L)=\sqrt{a K^{2}+b K L+c L^{2}},
$$

with $a, b, c \geq 0, a^{2}+b^{2}+c^{2} \neq 0$, then

$$
\mathcal{C}(z, \zeta)=\left(a K^{2}+b K L+c L^{2}\right)^{\frac{1}{4}}
$$

is a complex Cartan metric on the product manifold $M:=M_{1} \times M_{2}$. According to Theorem $4.3, \mathcal{C}$ is a purely Hermitian metric if and only if $b^{2}=4 a c$ and the complex Cartan metrics $K$ and $L$ are purely Hermitian.

So, considering two purely Hermitian complex Cartan metrics $K$ and $L$, the relation (4.5) with $a, b, c \geq 0$ and $b^{2} \neq 4 a c$, gives us examples of nonpurely Hermitian complex Cartan metrics. Moreover, these obtained metrics are Berwald-Cartan.

\section{References}

[1] M. Abate, G. Patrizio, Finsler Metrics - A Global Approach, Lecture Notes in Math., 1591, Springer-Verlag, 1994. 
[2] T. Aikou, Some remarks on locally conformal complex Berwald spaces, Finsler geometry (Seattle, WA, 1995), 109-120, Contemp. Math., 196, AMS Prov. RI, 1996.

[3] N. Aldea, G. Munteanu, On complex Landsberg and Berwald spaces, J. Geom. Phys., 62 (2012), 2, 368-380.

[4] N. Aldea, G. Munteanu, On complex Douglas spaces, J. Geom. Phys., 66 (2013), 80-93.

[5] N. Aldea, G. Munteanu, Recent results on complex Cartan spaces, manuscript 2013.

[6] N. Aldea, G. Munteanu, Some classes of complex Cartan spaces, Rev. Roumaine Math. Pures Appl. 57 (2012), 1, Tome LV, 5-15.

[7] M. Anastasiei, Geometry of Berwald-Cartan spaces, BSG Proc. 11, 1-9, Geom. Balkan Press, 2004.

[8] D. Bao, S.S. Chern, Z. Shen, An Introduction to Riemannian Finsler Geom., Graduate Texts in Math., 200, Springer-Verlag, 2000.

[9] J.J. Faran, The equivalence problem for complex Finsler Hamiltonians, Finsler geometry (Seattle, WA, 1995), 133-144, Contemp. Math., 196, Amer. Math. Soc., Providence, RI, 1996.

[10] S. Kobayashi, Invariant distances on complex manifolds and holomorphic mappings, J. Math. Soc. Japan, 19 (1967), 460-480.

[11] L. Kozma, I.R. Peter, H. Shimada, On the twisted product of Finsler manifolds, Reports on Math. Phys., 57, 3, 2006, 375-383.

[12] R. Miron, Hamilton Geometry, An. St. Univ. Al.I. Cuza, Iasi, 35 (1989), 38-85.

[13] R. Miron, D. Hrimiuc, H. Shimada, S. Sabau, The Geometry of Hamilton and Lagrange Spaces, Kluwer Acad. Publ., 118, FTPH, 2001.

[14] G. Munteanu, Complex spaces in Finsler, Lagrange and Hamilton geometries, Kluwer Acad. Publ., 141, FTPH, 2004.

[15] G. Munteanu, On Kobayashi dual metric, Libertas Math., 24 (2004), 6370 .

[16] Z. Shen, Differential Geometry of Spray and Finsler Spaces, Kluwer Academic Publishers, Dordrecht, 2001. 
[17] P.M. Wong, Theory of Complex Finsler Geometry and Geometry of Intrinsic Metrics, Imperial College Press 2011.

[18] W. Zhicheng, C. Zhong, Some results on product complex Finsler manifolds, Acta Math. Sci. Ser. B Engl. Ed. 31 (2011), no. 4, 1541-1552.

Nicoleta ALDEA,

Department of Mathematics and Computer Science,

Transilvania University of Brasov,

Iuliu Maniu 50, 500091 Brasov, Romania.

Email: nicoleta.aldea@lycos.com

Gheorghe MUNTEANU,

Department of Mathematics and Computer Science,

Transilvania University of Brasov,

Iuliu Maniu 50, 500091 Brasov, Romania.

Email: gh.munteanu@unitbv.ro 